\section{Radiosensitization of Glioma Cells by Temozolomide (TMZ): A Colony Formation Assay}

\author{
Somayyeh Babaloui ${ }^{1}$, Masoud Najafi², Hossein Mozdara- \\ $\mathrm{ni}^{3}$, Samira Borhani ${ }^{4}$, Ramin Jaberi5, Mahdi Aghili6*๑
}

\begin{abstract}
Background: Glioblastoma is one of the most radioresistant cancers. It is suggested that combination of radiotherapy with other cancer treatment modalities may increase control of tumor. Temozolomide (TMZ) is one of the most known drugs for glioblastoma. It has shown that TMZ via induction of mutation and cell death can kill glioma cells.

Objective: In the current study, we aimed to show possible radiosensitization effect of TMZ for glioma cells. In addition, results compared to response of normal fibroblast cells to TMZ and irradiation.

Material and Methods: This is in vitro study for evaluation of the effect of TMZ and irradiation on high grade glioma cells and normal fibroblasts. The human fibroblast and glioma cells were cultured as monolayer. The cells were treated with $2000 \mu \mathrm{M} \mathrm{TMZ}$, which was equal drug dose for IC50\%. In addition, irradiation was done with 5 Gy gamma rays. The formation of colony was observed following irradiation, treatment with TMZ, and combination both of them.
\end{abstract}

Results: The formation of colony for both glioma and fibroblast cells showed a reduction following irradiation or treatment with TMZ. Irradiation showed more toxicity compared to TMZ for glioma cells, but not fibroblast cells. Combination of TMZ and irradiation showed a significant reduction in the colony formation compared to irradiation or TMZ treatment alone.

Conclusion: This study showed that TMZ increases sensitivity of both glioma and fibroblast cells to ionizing radiation.

Citation: Babaloui S, Najafi M, Mozdarani H, Borhani S, Jaberi R, Aghili M. Radiosensitization of Glioma Cells by Temozolomide (TMZ): A Colony Formation Assay. J Biomed Phys Eng. 2022;12(1):43-50. doi: 10.31661/jbpe.v0i0.1223.

\section{Keywords}

Temozolomide; Radiation; Glioblastoma; Fibroblast Cells

\section{Introduction}

lioblastoma multiforme (GBM) is one of the most resistant hu-
man tumors that can originate from astrocytes within brain or
spinal cord [1]. Patients with GBM has poor survival [2]. Con-
trol of growth of glioblastoma is very difficult and usually complete
treatment isn't possible [3]. Radiotherapy and chemotherapy are the
most common modalities for reduction of cancer cells survival [4]. This
can reduce tumor growth, thus increase survival of patients [5]. Radio-
therapy is able to kill cells via direct interaction with genomic content
${ }^{1} \mathrm{PhD}$, Department of Medical Physics and Biomedical Engineering, School of Medicine, Tehran University of Medical Sciences, Tehran, Iran

${ }^{2} \mathrm{PhD}$, Department of Radiology and Nuclear Medicine, School of Paramedical Sciences, Kermanshah University of Medical Sciences, Kermanshah, Iran

${ }^{3} \mathrm{PhD}$, Department of Medical Genetics, Faculty of Medical Sciences, Tarbiat Modares University, Tehran, Iran

${ }^{4} \mathrm{MSc}$, Department of Radiology, Shahid Beheshti University of Medical Sciences, Tehran, Iran

${ }^{5} \mathrm{PhD}$, Radiation Oncology Research Center (RORC), Yas Radiation Oncology Center, Tehran University of Medical Sciences, Tehran, Iran

${ }^{6} \mathrm{MD}$, Cancer Research Center and Radiation Oncology Research Center (RORC), Iran Cancer Institute, Tehran University of Medical Sciences, Tehran, Iran

*Corresponding author: Mahdi Aghili

Cancer Research Center and Radiation Oncology Research Center (RORC), Iran Cancer Institute, Tehran University of Medical Sciences, Tehran, Iran

E-mail: aghili@tums.ac.ir

Received: 19 July 2019

Accepted: 13 August 2019 
of cells, and also through radiolysis of water molecules. Free radical production plays a main role for killing cells following exposure to radiation [6]. Because of severe toxicity of radiation for adjacent normal tissues, tumors can receive only a limited dose of radiation. Thus, it is necessary that patients receive another adjuvant for more suppression of tumor growth [7].

Temozolomide (TMZ) is an alkylating agent chemotherapy drug, approved by the food and drug administration (FDA) for some brain tumors, including glioblastoma [8]. It can be administrated orally because of its stability within acid environment [9]. Furthermore, because of small size and lipophilic properties of TMZ, it can pass from blood-brain barrier (BBB) easily [10]. It is suggested that anticancer activity of TMZ is different from ionizing radiation. It seems that $\mathrm{TMZ}$ can affect DNA directly by post-translational modifications. Moreover, TMZ can cause methylation of histone $\mathrm{H} 3$ protein and purine bases in DNA, leading to suppression of glioma cells proliferation [11].

In current study, we aimed to determine radiosensitization effect of TMZ in glioma cells. We evaluated the effect of TMZ combination with ionizing radiation on colony formation of glioma cells. Furthermore, we evaluated the toxic effect of this combination of the normal fibroblast cells.

\section{Material and Methods}

This is in vitro study for evaluation of the effect of TMZ and irradiation on high grade glioma cells and normal fibroblasts.

\section{Cell Lines}

U87MG human glioma cell line (ATCC ${ }^{\circledR}$ HTB-14 ${ }^{\mathrm{TM}}$ ) was provided by the cell bank of the Pastor Institute of Iran and fibroblast cell line of primary culture from the eyelid skin biopsy of a 45-year-old woman. Cells were maintained in Dulbecco's modified eagle's medium (DMEM, GIBCO), containing 10\% fetal bovine serum (FBS, GIBCO), $100 \mathrm{U} /$ $\mathrm{ml}$ penicillin (Sigma), $100 \mathrm{U} / \mathrm{ml}$ streptomycin (Sigma), and $2 \mathrm{mM} \mathrm{L-glutamine.}$

\section{Monolayer Cell Culture}

Both cell lines were cultured as monolayer at a cell density of $10^{4} \mathrm{cell} / \mathrm{cm}^{2}$ in T-25 flasks (Nunc). Cultures were maintained at $37^{\circ} \mathrm{C}$ in a humidified atmosphere of $5 \% \mathrm{CO}_{2}$. Cultures were propagated and cells were harvested by trypsinizing cultures with $1 \mathrm{mM}$ EDTA/ $0.25 \%$ Trypsin (w/v) (SIGMA) in Phosphate Buffer Saline (PBS).

\section{Drug Treatment with TMZ}

In order to evaluate the radiosensitizing effect of TMZ on glioma and primary fibroblast cells in the presence of irradiation (IR); 2000 $\mu \mathrm{M}$ of TMZ were added to the plates. Based on our previous study, this is the minimum concentration of TMZ in which cell growth is reduced by $50 \%$ (IC50) [12]. The plates contained monolayer cells with $2 \times 10^{3}$ cell $/$ $\mathrm{cm}^{2}$ density. Mentioned concentration was acquired with a solution of $2.5 \mathrm{mg}$ TMZ powder in $1 \mathrm{ml}$ of $0.2 \%$ DMSO in ambient temperature and kept at $-20{ }^{\circ} \mathrm{C}$. Samples were maintained under drug treatment for a cell doubling time (27 hours).

\section{Irradiation Treatment}

Glioma and primary fibroblast cells were treated with TMZ 27 hours before being exposed to ionizing radiation. The concentration of the chemical was $2000 \mu \mathrm{M}$, in MEM containing $10 \%$ FBS. After the treatment time, the medium containing drugs was removed and the cultures were washed 3 times with PBS and the cells were immediately irradiated using ${ }^{60} \mathrm{Co}$ source (Theratron 780, Canada) at a dose rate of $116.76 \mathrm{cGy} / \mathrm{min}$ for $5 \mathrm{~Gy}$. For radiation treatment, culture flasks were put under collimator of equipment at $80 \mathrm{~cm}$ distance of the head at ambient temperature, and the field size and the period of irradiation were $20 \times 20 \mathrm{~cm}^{2}$ and $4.31 \mathrm{~min}$, respectively. 
Radiosensitization Glioma Cells Temodal

Colony formation assay

After drug treatment and irradiation, single cells were plated for colony formation tests. In day 10 after plating, cultures were washed 3 times with PBS, fixed with $2 \%$ formaldehyde in PBS for 15 min and stained with $0.5 \%$ crystal violet; in addition, colonies were counted by invert microscope (Olympus).

In order to evaluate the ability of cells to form colonies, different concentrations of individual cells $(50,150,250,500$ cells) from spheroid seeded into $60 \mathrm{~mm}$ were plated with $10 \mathrm{ml}$ of DMEM supplemented with $10 \%$ FBS. Plating efficiency (PE) and surviving fraction (SF) were calculated, using the following equations:

$\operatorname{PE}(\%)=\frac{\text { the number of colonies }}{\text { the number of seeded cells }} \times 100$

$\mathrm{SF}=\frac{\text { the number of colonies }}{\text { the number of seeded cells } \times \% \mathrm{PE}}$

\section{Experimental Design}

In order to evaluate the effects of TMZ and IR on U87MG and skin fibroblast cell lines, growth curve was drawn in semi-logarithmic scale with cell numbers versus time during 12 days. For the combined treatment based on IC50 of TMZ, a concentration of approximately $2000 \mu \mathrm{M} / \mathrm{ml}$ was used. Therefore, the concentration of TMZ in the last step was con- sidered as $2000 \mu \mathrm{M}$ in order to achieve the desirable effect of less TMZ dose in combination with irradiation. The groups were treated with radiation and TMZ were divided as follows:

1. Control: received neither drug nor radiation

2. Control solvent: received neither drug nor radiation, but received $0.2 \%$ DMSO

3. TMZ: treated with $2000 \mu \mathrm{M}$ dose of TMZ

4. Co: treated with 5 Gy of Co-60 gammarays

5. TMZ+IR: treated with TMZ $(2000 \mu \mathrm{M})$ in combination with 5 Gy of Co-60 gamma-rays.

\section{Statistical Analysis}

Results were analyzed using SPSS version 16.0 and Excel 2010 software and data were obtained with triplicate experiments. The significance level of the data was compared by one-way ANOVA and Kruskal-Wallis nonparametric tests. A value of $\mathrm{p} \leq 0.05$ was considered statistically significant.

\section{Results}

Figure 1a and $\mathrm{b}$ show the U87MG cell line and human skin Fibroblast cells in the monolayer culture model. The growth curve of U87MG cells was drawn in semi-logarithmic scale with number of cells versus time during 12 days. By calculating the slope of the curve
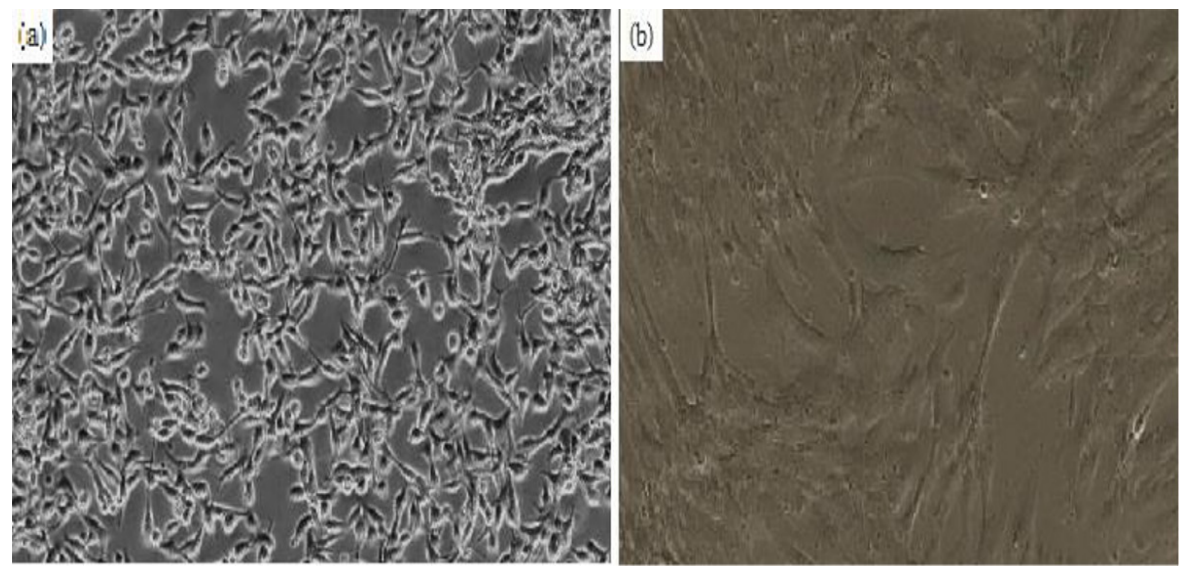

Figure 1: (a) U87MG cell line and (b) skin Fibroblast cells in the monolayer culture with 10X magnification. 
in logarithmic region, the doubling time (DT) of U87MG cell growth was obtained which was equal to $27.36 \pm 0.89$ hours.

The groups that need to be treated with TMZ were subjected to it for one doubling time, i.e. 27 hours, to initiate apoptosis process. Then all the groups were transferred to the radiotherapy department for irradiation. Finally, after drug and irradiation treatment, the number of colonies was counted for the evaluation of radiosensitization effects of TMZ. The average of maximum and minimum numbers of forming colonies were 112, 29 and 96, 33 for U87MG and Fibroblast cells, respectively.
Based on the results of colony formation of different numbers of pre-cultured cells, the optimum cell number to create colonies from single cells of U87MG was $\sim 150$ per $60 \mathrm{~mm}$ dishes. The results are shown clearly in Figure 2.

To ensure that any observed reduction in the survival fraction of the monolayer culture cells is not related to the immediate death of the cells based on staining them with TrypanBlue dye, the percentage of alive cells were immediately determined after the exposure. According to Figure 3, the percentage of alive cells in all treated groups were more than $95 \%$.

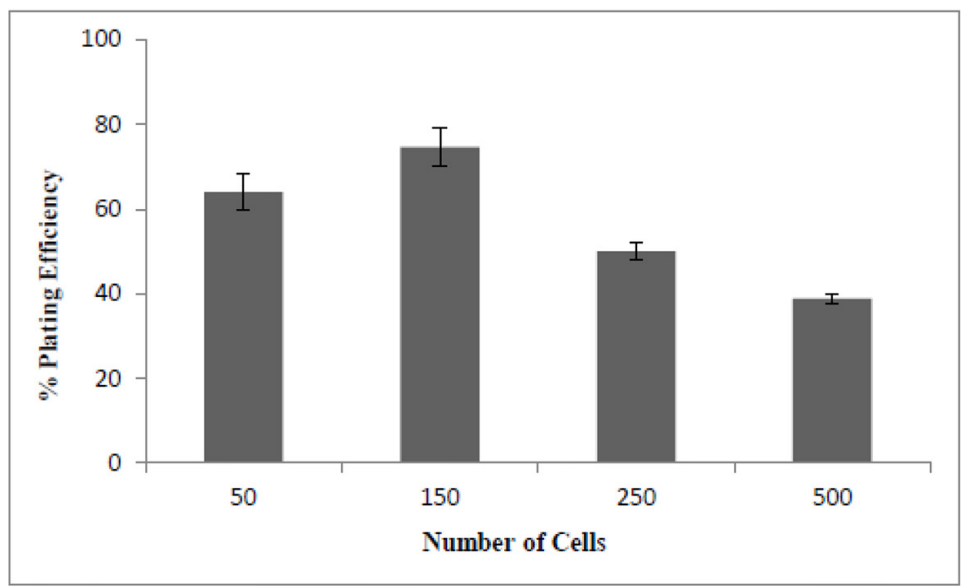

Figure 2: Plating efficiency using U87MG cells of monolayer culture in Dulbecco's modified eagle's medium (DMEM), supplemented with $10 \%$ fetal bovine serum (FBS) in different concentrations. Mean \pm Standard Error of the Mean (SEM) of 3 experiments.

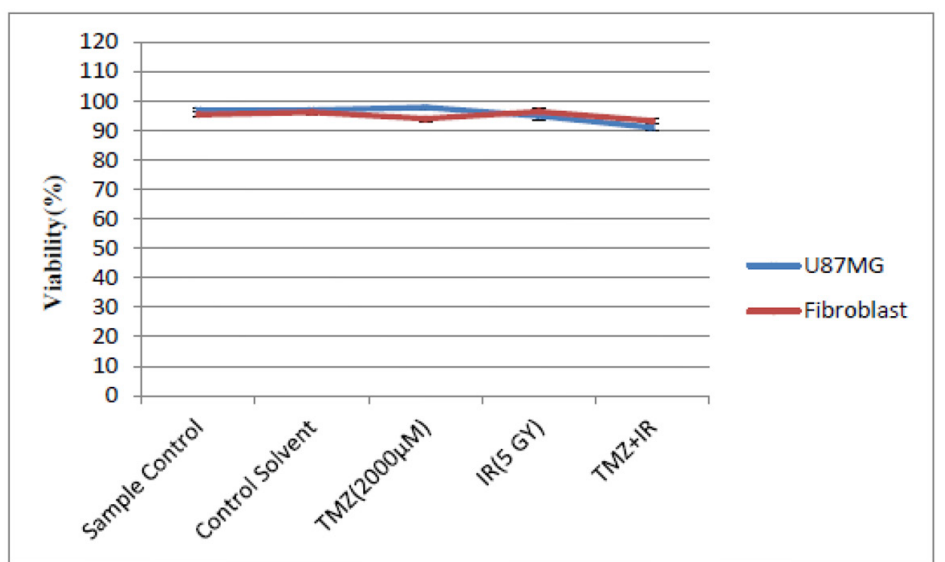

Figure 3: The percentage of living cells in different treated groups of U87MG and Fibroblast cells. Mean \pm Standard Error of the Mean (SEM) of 3 experiments. 
Radiosensitization Glioma Cells Temodal

Eventually, after exposing of samples by ${ }^{60} \mathrm{Co}$ in combination with TMZ, the ability of cells to form colonies was assessed using colony assay. Tables 1 and 2 show the number of colonies and PE percentage of cells in control and experimental groups under TMZ and IR treatment in both cell types.
As shown in Figure 4, the number of colonies counted in treated and irradiated samples was less than the control group. In addition, there were no statistical differences of data obtained for the $\% \mathrm{PE}$ between the control and control solvent groups $(\mathrm{P}=0.2$ and 0.4$)$, meaning $0.2 \%$ DMSO does not cause any cytotoxic

Table 1: A summary of data obtained for U87MG cells in treated samples. Data shows mean values from triplicate experiments. SD is the standard deviation of mean values.

\begin{tabular}{ccccc} 
Groups & Mean No of colonies & SD & Mean PE\% & SD \\
\hline Control & 112.33 & 6.03 & 74.88 & 4.02 \\
\hline Control solvent & 105.33 & 8.50 & 70.22 & 5.67 \\
\hline TMZ & 78.33 & 4.04 & 52.22 & 2.69 \\
\hline IR & 57.33 & 3.81 & 38.22 & 2.34 \\
\hline TMZ+IR & 29 & 3.00 & 19.33 & 2.00
\end{tabular}

SD: Standard deviation, PE: Plating efficiency, TMZ: Temozolomide, IR: Irradiation

Table 2: A summary of data obtained for primary fibroblast cells in treated samples. Data shows mean values from triplicate experiments. SD is the standard deviation of mean values.

\begin{tabular}{ccccc} 
Groups & Mean No of colonies & SD & Mean PE\% & SD \\
\hline Control & 96.33 & 5.03 & 64.22 & 3.36 \\
\hline Control solvent & 95.66 & 3.06 & 63.77 & 2.04 \\
\hline TMZ & 70 & 5.57 & 46.66 & 3.71 \\
\hline IR & 60.33 & 7.09 & 40.22 & 4.73 \\
\hline TMZ+IR & 33.66 & 4.16 & 22.66 & 2.77
\end{tabular}

SD: Standard deviation, PE: Plating efficiency, TMZ: Temozolomide, IR: Irradiation

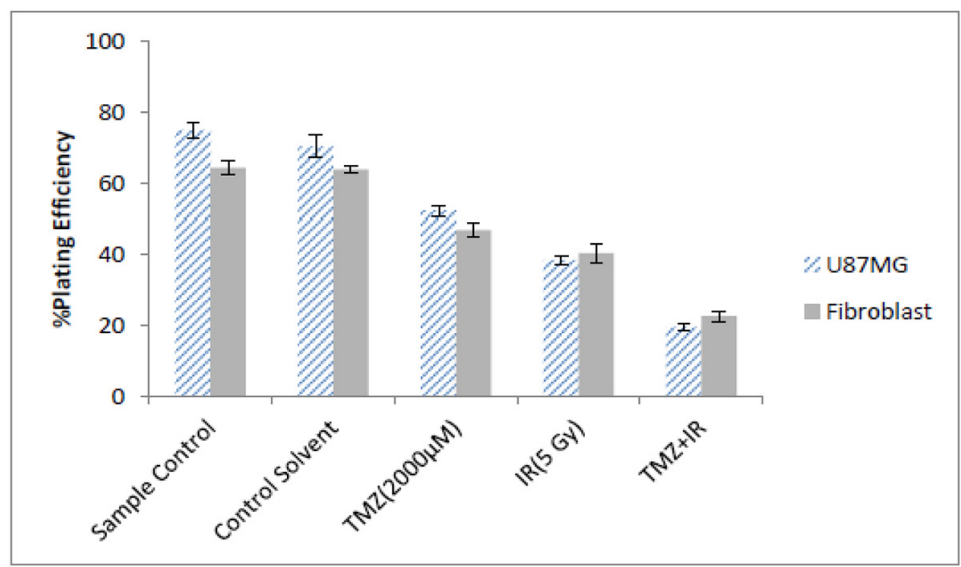

Figure 4: Plating efficiency using U87MG and fibroblast cells of monolayer culture in Dulbecco's modified eagle's medium (DMEM) supplemented with $10 \%$ fetal bovine serum (FBS) in different treated groups. Mean \pm Standard Error of the Mean (SEM) of 3 experiments. 
effect. Moreover, the difference between IR alone and $\mathrm{TMZ}$ alone for fibroblast cells was not statistically significant $(\mathrm{P}=0.5)$. However, for glioma cells, results showed a significant difference $(\mathrm{p}<0.05)$.

Treated group with TMZ+IR in both cell lines was compared to TMZ alone which showed a significant difference in U87MG and fibroblast $(\mathrm{P}=0.002$ and 0.001 respectively). Beside, treated group with $\mathrm{TMZ}+\mathrm{IR}$ in U87MG and fibroblast cells presented a significant difference compared to IR alone $(\mathrm{P}=0.009$ and 0.03 respectively).

There was also a big difference, in TMZ+IR in comparison with the control as well as other groups $(\mathrm{p}<0.002$ and $<0.03$ for U87MG and fibroblast respectively). In order to acquire the cytotoxic effect of TMZ on the cells in the presence of gamma radiation, the percentage difference of mean $\% \mathrm{PE}$ in $\mathrm{TMZ}+\mathrm{IR}$ relative to the mean $\% \mathrm{PE}$ in IR exposed group was evaluated. This discrepancy was $50 \%$ and $44 \%$ for U87MG and fibroblast, respectively.

Furthermore, according to Figure 5 that shows the survival fraction in both cell lines, the lowest survival fraction was observed in the combination of TMZ and IR that was $25 \%$ for $\mathrm{U} 87 \mathrm{MG}$ and $34 \%$ for fibroblast cells. In addition, there was no significance difference between the results obtained for U87MG cells and primary fibroblast $(\mathrm{P} \geq 0.05)$. All the results were in agreement with our results published before [12].

\section{Discussion}

TMZ is known as one of the most famous drugs for temporary reduction of tumor bulk [13]. It has been reported that TMZ via induction of mutation induces death in glioma cancer cells [13]. Furthermore, it has known that $\mathrm{TMZ}$ is able to induce hypermethylation in the promotor of DNA mismatch repair (MMR) genes, thus causes accumulation of unrepaired DNA damage and finally cell death [14]. For first time, in 2005, a clinical trial study showed that TMZ in combination with radiotherapy could increase survival of patients with glioblastoma significantly. Besides, this study showed that the toxicity for combination of TMZ with radiotherapy is minimal [15]. This lead to approval of TMZ by FDA for patients with newly diagnostic glioblastoma [16]. TMZ also has shown promising results for patients with high-grade glioblastoma cancer $[17,18]$. In recent years, a clinical trial study showed that TMZ is more useful for patients with methylated O6-methylguanine-DNA methyltransferase (MGMT) [4].

In the current study, we evaluated sensitization effect of TMZ in combination with radia-

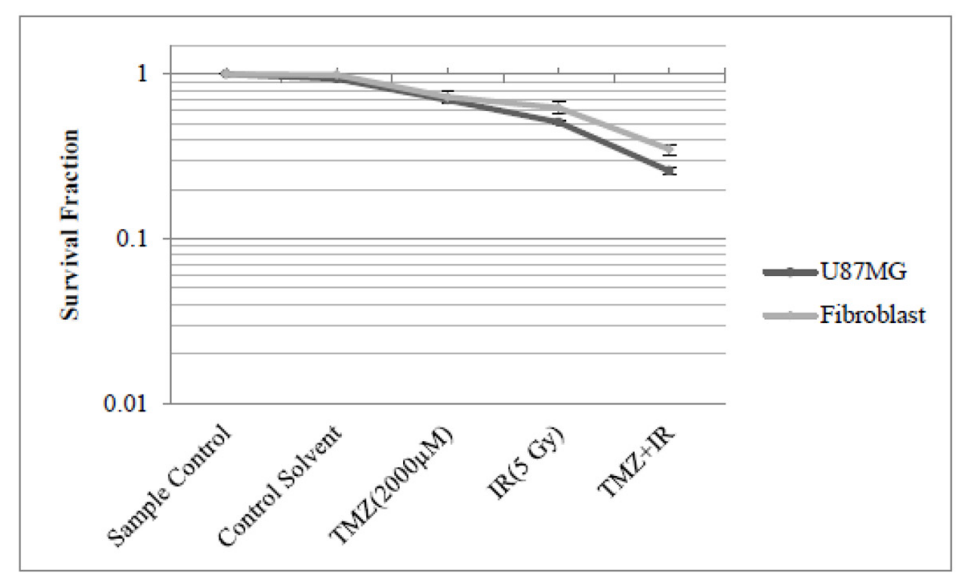

Figure 5: Survival fraction using U87MG and fibroblast cells of monolayer culture in Dulbecco's modified eagle's medium (DMEM) supplemented with $10 \%$ fetal bovine serum (FBS) in different treated groups. Mean \pm Standard Error of the Mean (SEM) of 3 experiments. 
Radiosensitization Glioma Cells Temodal

tion for malignant glioma and normal fibroblast cells. At the first level, our results showed that appropriated concentration of glioma cells for colony formation is 150 per $60 \mathrm{~mm}$ dishes. Lower or higher concentrations showed less efficiency. For evaluating the toxicity of TMZ on both normal fibroblast and glioma cells, we measured colony formation following treatment with TMZ. Our results showed that TMZ has a significant toxicity and causes the reduction of colony formation by more than $25 \%$ for both fibroblast and glioma cells. Results of $\mathrm{PE} \%$ showed no difference between glioma and fibroblast cells following treatment with $\mathrm{TMZ}$ as alone. Irradiation of glioma cells with a single dose of 5 Gy showed more efficiency compared to TMZ. Irradiation could reduce colony formation near to half of glioma cells. However, this reduction for fibroblast cells was not significant. Combination of TMZ with irradiation showed a remarkable reduction of colony formation and $\mathrm{PE} \%$. Results for both fibroblast and glioma cells presented a significant reduction compared to irradiation alone without treatment with TMZ. These results showed a significant radiosensitization effect of TMZ for glioma and fibroblast cells. Results of this study showed that TMZ might suppression of colony formation sensitize glioma cancer cells to radiation treatment.

\section{Conclusion}

In the current study, we show the effect of TMZ as a radiosensitizer using the evaluation of colony formation assay. Furthermore, the effect of both ionizing radiation and TMZ was alone determined. Results demonstrated a significant reduction of colony numbers following irradiation or treatment with TMZ. Irradiation showed more toxicity compared to TMZ alone for glioma cells, but not for fibroblast cells. Furthermore, the combination form of TMZ and irradiation was more toxic compared to treatment or irradiation alone. In conclusion, TMZ showed significant radiosensitization effects for glioma and fibroblast cells via reduction of colony formation.

\section{Acknowledgment}

The authors are grateful to the Ophthalmic Research Center of Iran for providing the radiobiology lab and also Cancer institute of Imam Khomeini Hospital for facilitating the irradiations. We also would like to thank Dr. Sahar Balagholi for her kind contribution in the study.

\section{Authors' Contribution}

Somayyeh Babaloui, Ramin Jaberi and Hossein Mozdarani investigated and conceived the idea. The original draft of the paper was written by Somayyeh Babaloui and Masoud Najafi. Samira Borhani gathered the images and the related literature and also helped with writing of the related works. The method implementation was carried out by Samira Borhani. Results and Analysis was carried out by Somayyeh Babaloui, Masoud Najafi and Samira Borhani. The Project administration was performed by Mahdi Aghili and supervised by Ramin Jaberi. All the authors reviewed, modified, and approved the final version of the manuscript.

\section{Funding}

This project was funded by Cancer Research Institute, Tehran University of Medical Sciences, Tehran, Iran.

\section{Conflict of Interest}

None

\section{References}

1. Nagaishi M, Nobusawa S, Yokoo H, Sugiura $Y$, Tsuda $\mathrm{K}$, et al. Genetic mutations in high grade gliomas of the adult spinal cord. Brain Tumor Pathology. 2016;33(4):267-9. doi: 10.1007/s10014016-0263-7.

2. Delgado-López PD, Corrales-García EM. Survival in glioblastoma: a review on the impact of treatment modalities. Clin Trans/ Oncol. 2016;18(11):106271. doi: 10.1007/s12094-016-1497-x. PubMed PMID: 26960561.

3. Jahani-AsI A, Yin H, Soleimani VD, Haque T, Luchman HA, Chang NC, et al. Control of glioblastoma tumorigenesis by feed-forward cytokine signaling. Nat Neurosci. 2016;19(6):798-806. doi: 10.1038/ nn.4295. PubMed PMID: 27110918. PubMed PMCID: PMC4984527. 
4. Perry JR, Laperriere N, O'Callaghan CJ, Brandes AA, Menten J, Phillips C, et al. Short-course radiation plus temozolomide in elderly patients with glioblastoma. N Engl J Med. 2017;376(11):102737. doi: 10.1056/NEJMoa1611977. PubMed PMID: 28296618.

5. Haque W, Verma V, Butler EB, Teh BS. Addition of chemotherapy to hypofractionated radiotherapy for glioblastoma: practice patterns, outcomes, and predictors of survival. $J$ Neurooncol. 2018;136(2):307-15. doi: 10.1007/s11060-0172654-y. PubMed PMID: 29090416.

6. Song G, Cheng L, Chao Y, Yang K, Liu Z. Emerging nanotechnology and advanced materials for cancer radiation therapy. Adv Mater. 2017;29(32). doi: 10.1002/adma.201700996. PubMed PMID: 28643452.

7. Siddiqui F, Movsas B. Management of radiation toxicity in head and neck cancers. Semin Radiat Oncol. 2017;27(4):340-9. doi: 10.1016/j.semradonc.2017.04.008. PubMed PMID: 28865517.

8. Tateishi K, Higuchi F, Miller JJ, Koerner MV, Lelic $\mathrm{N}$, Shankar GM, et al. The alkylating chemotherapeutic temozolomide induces metabolic stress in IDH1-mutant cancers and potentiates NAD+ depletion-mediated cytotoxicity. Cancer Res. 2017; 77(15):4102-15. doi: 10.1158/0008-5472.CAN-162263. PubMed PMID: 28625978. PubMed PMCID: PMC5783559.

9. Rao JU, Coman D, Walsh JJ, Ali MM, Huang Y, Hyder F. Temozolomide arrests glioma growth and normalizes intratumoral extracellular $\mathrm{pH}$. Scientific Reports. 2017;7(1):1-6. doi: 10.1038/s41598-01707609-7.

10. Kumari S, Ahsan SM, Kumar JM, Kondapi AK, Rao NM. Overcoming blood brain barrier with a dual purpose Temozolomide loaded Lactoferrin nanoparticles for combating glioma (SERP-1712433). Sci Rep. 2017;7(1):6602. doi: 10.1038/ s41598-017-06888-4. PubMed PMID: 28747713. PubMed PMCID: PMC5529576.

11. Wang T, Pickard AJ, Gallo JM. Histone methylation by temozolomide; a classic DNA methylating anticancer drug. Anticancer Res. 2016;36(7):328999. PubMed PMID: 27354585. PubMed PMCID: PMC5349185.

12. Borhani S, Mozdarani H, Babalui S, Bakhshandeh $M$, Nosrati $H$. In vitro radiosensitizing ef- fects of temozolomide on U87MG cell lines of human glioblastoma multiforme. Iran J Med Sci. 2017;42(3):258-65. PubMed PMID: 28533574 PubMed PMCID: PMC5429494.

13. Daniel P, Sabri S, Chaddad A, Meehan B, JeanClaude B, Rak J, Abdulkarim BS. Temozolomide induced hypermutation in glioma: evolutionary mechanisms and therapeutic opportunities. Front Oncol. 2019;9:41. doi: 10.3389/fonc.2019.00041. PubMed PMID: 30778375. PubMed PMCID: PMC6369148.

14. Felsberg J, Thon N, Eigenbrod S, Hentschel B, Sabel MC, Westphal M, et al. Promoter methylation and expression of MGMT and the DNA mismatch repair genes MLH1, MSH2, MSH6 and PMS2 in paired primary and recurrent glioblastomas. Int $J$ Cancer. 2011;129(3):659-70. doi: 10.1002/ ijc.26083. PubMed PMID: 21425258.

15. Stupp R, Mason WP, Van Den Bent MJ, Weller M, Fisher B, Taphoorn MJ, Belanger K, Brandes AA, Marosi C, Bogdahn U, Curschmann J. Radiotherapy plus concomitant and adjuvant temozolomide for glioblastoma. N Engl J Med. 2005;352(10):98796. doi: 10.1056/NEJMoa043330. PubMed PMID: 15758009.

16. Cohen MH, Johnson JR, Pazdur R. Food and Drug Administration Drug approval summary: temozolomide plus radiation therapy for the treatment of newly diagnosed glioblastoma multiforme. Clin Cancer Res. 2005;11(19):6767-71. doi: 10.1158/1078-0432.CCR-05-0722. PubMed PMID: 16203762.

17. Parisi S, Corsa P, Raguso A, Perrone A, Cossa S, Munafò T, Sanpaolo G, Donno E, Clemente MA, Piombino M, Parisi F. Temozolomide and radiotherapy versus radiotherapy alone in high grade gliomas: a very long term comparative study and literature review. Biomed Res Int. 2015;2015:620643. doi: 10.1155/2015/620643. PubMed PMID: 25815327. PubMed PMCID: PMC4359808.

18. Mirimanoff R0, Gorlia T, Mason W, Van Den Bent MJ, Kortmann RD, Fisher B, Reni M, Brandes AA, Curschmann J, Villa S, Cairncross G. Radiotherapy and temozolomide for newly diagnosed glioblastoma: recursive partitioning analysis of the EORTC 26981/22981-NCIC CE3 phase III randomized trial. J Clin Oncol. 2006;24(16):2563-9. doi: 10.1200/ JC0.2005.04.5963. PubMed PMID: 16735709. 